\title{
Development of a fusion-oriented pulsed power module
}

\author{
Lin Chen, ${ }^{1}$ Wenkang Zou, ${ }^{1, *}$ Liangji Zhou, ${ }^{1}$ Meng Wang, ${ }^{1}$ Yu Liu, ${ }^{1}$ Laqun Liu, ${ }^{2}$ \\ Mingmeng Deng, ${ }^{3}$ Dagang Liu, ${ }^{2}$ Jianping Zhu, ${ }^{3}$ Kenan Lian, ${ }^{3}$ Bing Wei, ${ }^{1}$ Qing Tian, ${ }^{1}$ \\ Fan Guo, ${ }^{1}$ An He, ${ }_{1}^{1}$ Shuping Feng, ${ }^{1}$ Weiping Xie, ${ }^{1}$ Lin Meng, ${ }^{2}$ Jianjun Deng, ${ }^{1}$ \\ Yue Zhao, ${ }^{1}$ Le Zhang, ${ }_{1}^{1}$ Feng Li, ${ }_{1}$ Jihao Jiang, ${ }^{1}$ Jie Wang, ${ }^{1}$ Yuanjun Zhang, ${ }^{1}$ Yong Li, ${ }^{1}$ \\ Yingmin Dai, ${ }^{1}$ Wenhui Han, ${ }^{1}$ Ye Li, ${ }^{1}$ Bin Yao, ${ }^{1}$ Yu Ding, ${ }^{1}$ and Junjun Kang ${ }^{1}$ \\ ${ }^{1}$ Key Laboratory of Pulsed Power, Institute of Fluid Physics, \\ China Academy of Engineering Physics, Mianyang 621999, China \\ ${ }^{2}$ School of Electronic Science and Engineering, University of Electronic Science and Technology of China, \\ Chengdu 610054, China \\ ${ }^{3}$ Institute of Mechanical Manufacturing Technology, \\ China Academy of Engineering Physics, Mianyang 621999, China
}

(Received 17 December 2017; revised manuscript received 22 February 2019; published 13 March 2019)

\begin{abstract}
We present a fusion-oriented pulsed power module M-50, which is based on the linear transformer driver (LTD) and magnetically insulated inductive voltage adder (MIVA) technologies. The module M-50, which consists of 50 identical LTD cavities connected in series, is one of the 60 modules of a fusion-scale pulsed power facility. M-50 is a comprehensive test bed for LTD integration and engineering validation. Each cavity consists of 32 bricks and has an output capability of $90 \mathrm{kV} / 1.0 \mathrm{MA} / 120 \mathrm{~ns}$ to the matched load. The output power of the 50 cavities is added with a MIVA, whose operation impedance is approximately matched to both source and load. Therefore, it has a nominal output capability of 4.5 MV/1.0 MA/120 ns to $4.5 \Omega$ resistive load. The module is divided into five groups, and each group has ten cavities in series. The inner stalk of the MIVA is divided into five main straight segments. Conical transitions are employed to connect adjacent straight segments. The output end of M-50 is shrunk and connected a ring-cathode diode, whose cathode and anode radii are identical to those of a 12-m-long transmission line in the fusion facility. In this paper, the general concept of the fusion accelerator, the physical design, engineering design and development progress of M-50 are described for the first time.
\end{abstract}

DOI: 10.1103/PhysRevAccelBeams.22.030401

\section{INTRODUCTION}

Pulsed power driven inertial confinement fusion (ICF) has been regarded as one of the most promising approaches for controlled fusion in the past decade [1-6]. This scientific judgment was based on a series of physical breakthroughs [7] dated from the 1990s at Sandia National Laboratories, persistent progress in Z-pinch [8-11] and pulsed power technology around the world.

Pulsed power driven ICF utilizes the x-ray or kinetic energy from the electromagnetic implosion to compress the fusion capsule to achieve ignition condition. The advantages of this approach are high plug to x-ray or to kinetic energy efficiency, abundant in loading energy and low cost. Scaling relation indicates Z-pinch driven by $50-60$

\footnotetext{
*zwk@caep.cn

Published by the American Physical Society under the terms of the Creative Commons Attribution 4.0 International license. Further distribution of this work must maintain attribution to the author(s) and the published article's title, journal citation, and DOI.
}

mega-ampere (MA) current could realize thermonuclear ignition, in which the fusion yield exceeds the energy transmitted to the load [12-16]. Up to date, though the most powerful accelerators, ZR [17], Primary Test Stand (PTS) [18], Angara-5-1 [19], have successfully generated 5-26 MA current to physical packages, there is still a long distance ahead to fusion ignition.

$\mathrm{ZR}$ is based on the Marx and water-line technology, which has been proved to be very successful in construction MA/TW and beyond accelerators in the USA, Russia, and China in the past decades. In the Marx based scheme, each facility module will generate a current of 0.4-1 MA. This means that the main switch, for example the laser triggered gas switch (LTGS) [20,21] will suffer very large Coulomb and serious electrode erosion in every shot. Lifetime of the LTGS is estimated to be hundreds of shots, which prevents it being used in rep-rate drivers. Another concern for Marx technology is the energy loss in pulse formation and transmission. The output pulse from Marx is in $\mu s$ rising time. When it is temporally compressed to $\sim 100 \mathrm{ns,} \mathrm{a}$ majority of energy is dissipated to dielectrics. As a singleshot machine, comparing with other ICF approaches, an 
energy efficiency of $15 \%$ is very high. However, if working as a rep-rate facility, the energy dissipated into the system is very harmful. It may cause shocking damages or rise of component temperature. Therefore, pursuing other pulse driver technology has become an important issue for the pulsed power community in the past ten years [22,23].

The linear transformer driver (LTD) [24,25], which could directly produce much faster pulses than the conventional Marx generator, is one of the most promising alternatives. LTD uses low capacitance, low inductance components, and low jitter switches as basic discharge unit (i.e. brick), therefore, the rise time of output pulse from the basic discharging unit is as little as $100 \mathrm{~ns}$ or even faster. By synchronizing tens of such units and discharging to a common load, current up to MA could be generated by a single LTD cavity without any pulse compression. Furthermore, though the output voltage of a single cavity is usually no more than $100 \mathrm{kV}$, power could be summed up with voltage adder in a module and further converged by paralleling modules. Switches in LTD suffer much less current than the main switches in Marx-based facilities, thus one of the advantages in the LTD-based facility is longer lifetime and higher reliability. Meanwhile, eliminating pulse compression means higher energy transmission efficiency. Additionally, with a much larger component number, the final output pulse of the facility could be shaped with higher accuracy by controlling the trigger sequence of modules, cavities or even bricks within cavities. This makes LTD technology also attractive for dynamic material experiments [26], in which the current pulse is shaped to obtain a different current history.

LTD cavity with different parameters has been successfully developed around the world [27-39]. In some applications with $\sim 100 \mathrm{kA}$ current, for example, x-ray radiography, LTD cavities have been integrated into facilities [27,29,35-38]. Test results from these facilities indicated good performance. However, for fusion application, the MA current LTD cavity has more discharge bricks, much larger magnetic core, more charging and triggering cables, thus has much larger cavity diameter. This brings more challenges in engineering realization, especially in facility scale, such as dielectric sealing, inductive voltage adder (IVA) [40] stalk positioning, reliable trigger and synchronization, and daily maintaining. The Sandia scientists had done seminal work on the MYKONOS [34] facility in the direction of construction fusion accelerator, in which up to ten 1-MA LTD cavities were integrated with deionized water IVA. An output pulse of $1 \mathrm{MA} / 1 \mathrm{MV}$ is supposed to be obtained on load and more progress towards larger scale is expected on the way.

In this paper, development of a fusion-oriented pulsed power module, M-50, is reported for the first time. M-50 is based on LTD, magnetically insulated transmission line (MITL) [41-43] and IVA technology. It is a full-size module of a fusion scale pulsed power facility, and serves as a demonstration platform for related technology, such as premagnetization, charging, triggering, voltage adder, status monitoring and fault diagnostics, mechanics design and daily operation.

The remaining contents of this paper are arranged as follow. In Sec. II, the general concept of the fusion accelerator is briefly introduced. In Sec. III, the designs of M-50 and major subsystems are described. Engineering progress, including switch development, cavity test, trigger system, IVA system, are presented in Sec. IV. As conclusions, a brief summary and outlook to future work are presented in Sec. V.

\section{CONCEPT DESIGN OF FUSION SCALE DRIVER}

It is not exactly known what the optimized driving current is for fusion ignition, though a lot of remarkable works have been done in Z-pinch ICF. However, the Z-pinch and pulsed power communities have a common consciousness that $60 \mathrm{MA}$ or even $50 \mathrm{MA}$ current is very likely to fulfill ignition with a certain ignition scheme, for example, the latest suggested magnetized liner inertial fusion (MagLIF) concept $[5,15,16]$.

The coupling of pulse driver with fusion load is very complicated; however, as an input for pulsed power design, a target amplitude and rising time of current should be suggested. Without these parameters, technology demonstration could not be carried out. According to assessing a series of numerical simulation, and referring to some judgments from the worldwide community, a maximum current above $50 \mathrm{MA}$ and $10 \%-90 \%$ rising time of about 120 ns were chosen as target parameters for our concept design.

Figure 1 shows the schematic drawing of the facility concept. Sixty LTD modules in two layers are connected to current convergent structures via $12-\mathrm{m}$ coaxial MITLs. Fifty LTD cavities are used in each module and connected in series by a voltage adder. Each cavity consists of 32 bricks (one switch plus two capacitors), therefore, the facility consists of 3,000 LTD cavities, 96000 switches and 192000 capacitors in all. The nominal output of each module to a matched load is $4.5 \mathrm{MV} / 1 \mathrm{MA}$. The secondary of the voltage adder is insulated with vacuum, which has been proved very efficient in previous facilities. The vacuum could significantly reduce the insulation distance between electrodes, and avoid using lots of deionized water and the water-vacuum interface. MITL may introduce some current loss and pulse front erosion before magnetic insulation is fully established. However, this adverse effect could be compensated by setting the necessary margin in target current.

The 60 coaxial MITL are connected to conical disk MITLs after a transition section [44]. Each of the coaxial MITLs works at $4.5 \mathrm{MV}$ voltage and $1 \mathrm{MA}$ current, i.e. an operation impedance of $4.5 \Omega$. By paralleling the 60 lines, the effective impedance of coaxial MITLs is about $0.075 \Omega$. 


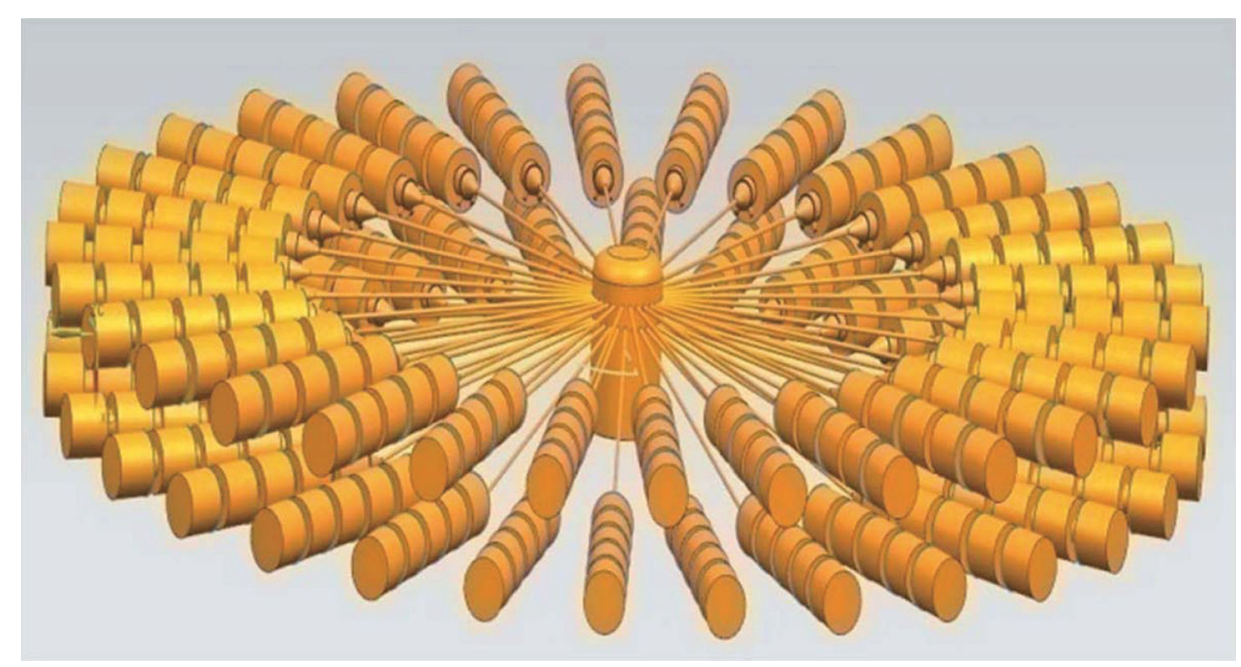

FIG. 1. Schematic drawing of the fusion facility concept. Sixty LTD modules in two layers are connected in parallel to current convergent structures where the fusion load is fielded to the end. In each module, 50 LTD cavities are connected with vacuum MIVA. The output of the module is connected to the conical transmission line via a 12-m coaxial MITL. The diameter and the height of the facility are about $65 \mathrm{~m}$ and $10 \mathrm{~m}$, respectively.

In order to avoid large current loss, the transition and the conical MITLs should have very low impedance. Low impedance in the final transmission lines is better matched with wire array or liner load. All the modules are supposed to be triggered with a reliable trigger unit according to optimized timing sequence, thus the LTD cavities in each module should be triggered with necessary delay in accordance with the speed of the electromagnetic wave in the IVA. Based on the testing experience of existing cavities, the output voltage of the trigger is at least $100 \mathrm{kV}$, with a maximum rising time of $30 \mathrm{~ns}$ and a jitter within 3 ns. Table I presents the main specification of the concept facility. Some of the cavity parameters will be further described in following sections.

The output current of the conceptual facility was estimated by circuit simulation, in which the 60 modules were lumped as a single module with 50 cavities. The static inductance of the convergent transmission line and wire array load behind the coaxial MITL was estimated to be $6.5 \mathrm{nH}$. A wire array load with initial radius of $2.0 \mathrm{~cm}$, height of $2.0 \mathrm{~cm}$ and mass of $220 \mathrm{mg}$ was imported with zero-dimensional (0D) implosion model. Figure 2 shows the obtained load current and implosion kinetic energy. The maximum current is about $62 \mathrm{MA}$ and the $10 \%-90 \%$ rising time is $120 \mathrm{~ns}$. The maximum kinetic energy from the implosion is $12.3 \mathrm{MJ}$.

In order to technically validate the concept, a step-bystep demonstration in LTD technology is essential. In the past decade, LTD cavity with MA current output capability was successfully proved. Even the voltage adding and power summations of several MA cavities were demonstrated on the MYKONOS facility. For a complete module in the fusion accelerator, when 50 cavities are connected, the system complexity and engineering difficulty will be much higher. However, a full-size module is an inevitable step on the road of technology development. For this purpose, a full-size module of the fusion facility, which is named M-50, is developed.

TABLE I. Major specifications of the concept design.

\begin{tabular}{lll}
\hline \hline System & \multicolumn{1}{c}{ Subsystem } & \multicolumn{1}{c}{ Descriptions } \\
\hline LTD cavities & Cavity & 32 bricks, $Z=0.09 \Omega, I_{\max } \sim 1.03 \mathrm{MA}, \tau_{r 10-90 \%} \sim 120 \mathrm{~ns}$ \\
& Switch & $V_{\text {hold-off }}=200 \mathrm{kV}, I>35 \mathrm{kA}, \mathrm{jitter}<2 \mathrm{~ns}, L<120 \mathrm{nH}$ \\
& Capacitor & $C=100 \mathrm{nF}, V_{\max }=100 \mathrm{kV}$ \\
MITL & Metglas magnetic core & Volt-second product $>28 \mathrm{mV} \cdot \mathrm{s}$ \\
& IVA & Magnetic insulation, matched to load, step segments, \\
& & $Z_{\text {op }}=0.09 \sim 4.5 \Omega$ \\
& Coaxial & $Z_{\text {op }}=4.5 \Omega$, length $=12 \mathrm{~m}$ \\
& Transition & Coaxial to conical \\
Trigger & Final MITL & PHC and two-electrode MITL \\
Charger & $\ldots$ & $V_{r}>100 \mathrm{kV}, \tau_{r}<30 \mathrm{~ns}$, jitter $<3 \mathrm{~ns}$ \\
\hline \hline
\end{tabular}




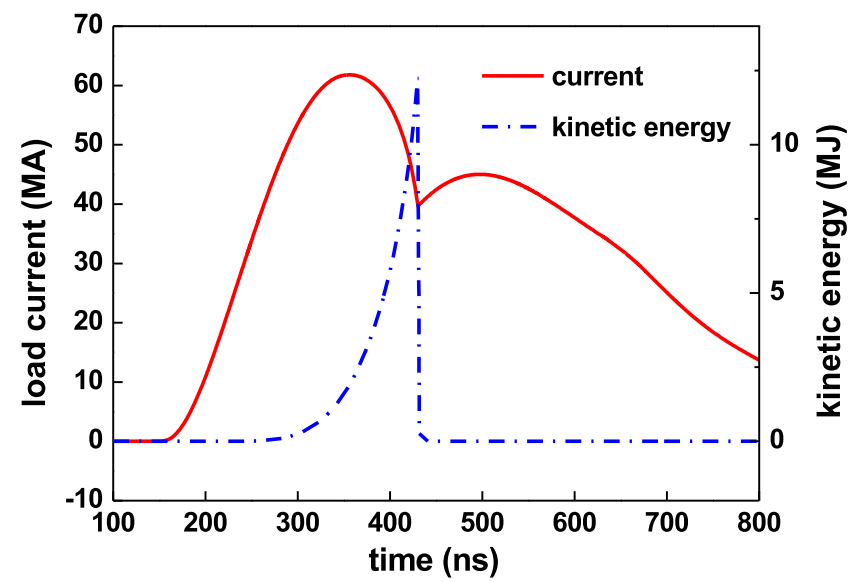

FIG. 2. Load current (red solid) and implosion kinetic energy (blue dash) from lumped circuit simulation for the concept design. The current and kinetic energy obtained on a Z-pinch load are $62 \mathrm{MA}$ and $12.3 \mathrm{MJ}$, respectively.

\section{THE PHYSICAL AND ENGINEERING DESIGN OF M-50}

\section{A. MIVA physical design}

In an IVA, the output voltage from individual power sources is summed up and the high voltage is restricted in the secondary, by penetrating an inner stalk through all sources. Each power source works as a one-to-one transformer [40]. Magnetic cores are used in the primary to prevent majority current loss to the ground, but flows in the secondary and finally to the load. For an ideal IVA, the impedance of the secondary should increase cavity by cavity. For M-50, the diameter of the outer electrode in IVA keeps constant; therefore, the diameter of the inner electrode should be decreased to accommodate voltage increasing cavity by cavity. This needs 50 stalk segments for 50 cavities, and makes fabrication and alignment very complex; because the whole inner stalk has a total length of about $20 \mathrm{~m}$, and the gravity induced deflections have great influence to the alignment. To make a compromise, the diameter of each cavity group keeps constant and only a single segment is used for ten cavities. Together with the transition and conjunction, only ten more segments are used for the whole inner stalk Fig. 3.

M-50 works in negative polarity. With several MV working voltage, the inner stalk of IVA (cathode) will suffer very high electric field, and explosive electron emission will take place within most of the length. Fortunately, the current is large enough to establish efficient magnetic insulation by the self-induced magnetic field. The diameter of each segment was determined according to the magnetic insulation theory and in such a way that the operation impedance in this group was matched to the final cavity in that group. The operation impedance of MITL was determined by its working voltage $V$ and current $I$. According to Creedon [42,43] and lots of further investigations, it is convenient to use the minimum magnetic insulation current $\left(I_{\min }\right)$ to estimate the operating impedance $[40,43]$. For the MITL segment with working voltage of $V_{a}$, the operating impedance $Z_{\mathrm{op}}$ has

$$
Z_{\mathrm{op}}=\frac{V_{a}}{I_{\min }}=\frac{V_{a}}{I_{0} g \gamma_{m}{ }^{3} \ln \left[\gamma_{m}+\left(\gamma_{m}{ }^{2}-1\right)^{1 / 2}\right]},
$$

where $I_{0}=8500 \mathrm{~A}$ is Alfen current, and $g$ is a geometrical factor, $\gamma_{m}$ is defined in terms of voltage $V_{m}$ by

$$
\gamma_{m}=1+\frac{e V_{m}}{m_{0} c^{2}}
$$

where $e$ and $m_{0}$ are charge and rest mass of an electron, $c$ is light speed in vacuum. Here, $\gamma_{m}$ is geometry independent and could be numerically obtained when anode voltage is given under minimum magnetic insulation current. Let

$$
x=I_{0} \gamma_{m}{ }^{3} \ln \left[\gamma_{m}+\left(\gamma_{m}{ }^{2}-1\right)^{1 / 2}\right],
$$

and from Eq. (1) one has

$$
Z_{\mathrm{op}}=\frac{V_{a}}{g x}
$$

For coaxial line, one has

$$
g=60 / Z_{v}
$$

Then the vacuum impedance

$$
Z_{v}=\frac{60 x Z_{\mathrm{op}}}{V_{a}} .
$$

The goal of our IVA design was to obtain a voltage of 4.5 MV and to drive a matched load of $4.5 \Omega$. Therefore, we set the target $V_{a}$ to be $0.9,1.8,2.7,3.6$, and $4.5 \mathrm{MV}$, and $Z_{\text {op }}$ to be $0.9,1.8,2.7,3.6$, and $4.5 \Omega$ for the five stalk segments. Then the vacuum impedance and cathode radius $R_{c}$ were determined. The parameters are listed in Table II.

Three-dimensional (3D) particle-in-cell (PIC) simulations were conducted to check the power flow by CHIPIC-3D [45], a full electromagnetic paralleled PIC code. Since the

TABLE II. Parameters of IVA segments. The anode radius $R_{a}$ is $600 \mathrm{~mm}$. The radius of the cathode segment is decreasing to accommodate impedance increasing. The operation impedance at the final cavity in each cavity group was matched to cavities.

\begin{tabular}{lccccc}
\hline \hline IVA segment & 1 & 2 & 3 & 4 & 5 \\
\hline$V_{a}(\mathrm{MV})$ & 0.9 & 1.8 & 2.7 & 3.6 & 4.5 \\
$Z_{\mathrm{op}}(\Omega)$ & 0.9 & 1.8 & 2.7 & 3.6 & 4.5 \\
$Z_{v}(\Omega)$ & 1.6 & 2.8 & 3.8 & 4.9 & 5.9 \\
$R_{c}(\mathrm{~mm})$ & 584 & 573 & 563 & 553 & 543 \\
\hline \hline
\end{tabular}




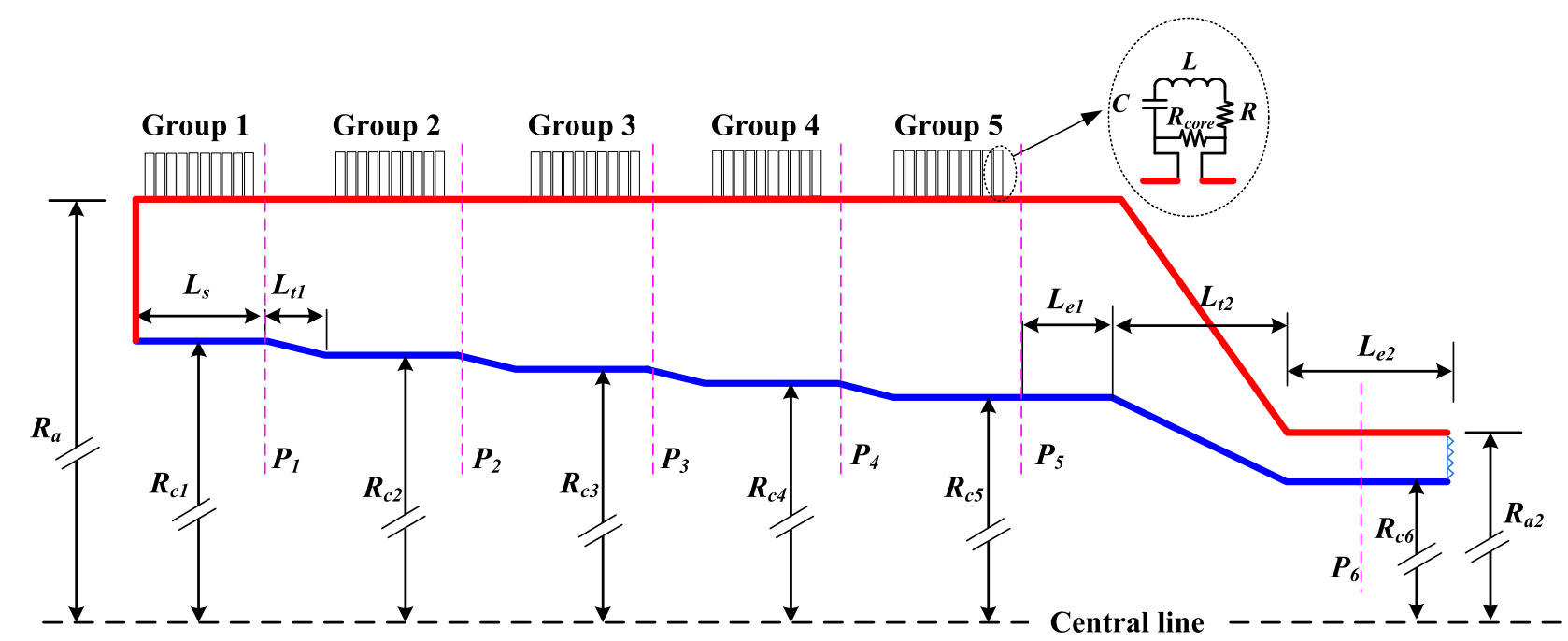

FIG. 3. Schematic of the model in PIC simulation. The external circuit boundary was used to drive the IVA. A conical section connects IVA to a coax in a smaller diameter. A matched output boundary was used at the end of the coax to avoid reflection. The lengths of final conical transition $\left(L_{t 2}\right)$ and the final coax $\left(L_{e 2}\right)$, and the load impedance were changed in different runs to achieve the different status of MITL operation.

total simulation length was more than $20 \mathrm{~m}$ and the MITL A-K gap was only a few $\mathrm{cm}$, it is very difficult and inefficient to carry out the computation in a single computer. The large aspect ratio of the geometry also made it impossible to use large cell, otherwise, large simulation error would generate. The method we used was dividing the IVA into many areas and computing each area with a computer, and the computers worked in parallel. In this way, the aspect ratio for each area was much smaller. At every time step, data from each computer were exchanged at the interface of adjacent areas.

A simple RLC circuit was imported into the model for each power port. The equivalent values of capacitance, inductance, and resistance were identical with that of a LTD cavity, which will be presented in the following content. An incident wave was generated and injected to the PIC boundary, at the same time, the reflected wave from IVA could transmit back to circuit [46]. A matched output boundary was used at the end of the coax to avoid reflection. In such a load boundary, the MITL worked in a self-limited flow.

Figures 4-6 show the voltage, anode current and cathode current observed at planes $P_{1}$ to $P_{6}$ from the simulation. For the presented case, the operating point was not at the minimum current point, but results show voltage could be effectively summed up and a maximum value of 4.6 MV could be obtained at the final MITL. The maximum current after each group was above 1.1 MA, which was also slightly higher than the designed value. However, the almost constant anode current indicates magnetic insulation was well maintained. As voltage went higher, the cathode current declined when approaching load, which indicates more and more current became electron current and flew in the anode-cathode gap.

\section{B. LTD cavity design $[47,48]$}

The cavity in M-50 each has 32 bricks electrically paralleled and in spoke layout (Fig. 7). Each brick consists of two $100 \mathrm{nF}$ capacitors, and a multi-gap gas switch, which has a maximum hold-off voltage of $200 \mathrm{kV}$. When placed in the cavity, the equivalent inductance and resistance of a brick are about $300 \mathrm{nH}$ and $0.25 \Omega$, respectively. The 32 switches in a cavity are triggered by four trigger pulses, each pulse corresponding to eight switches. The nominal energy storage of a cavity is $32 \mathrm{~kJ}$ at $100 \mathrm{kV}$ charging voltage.

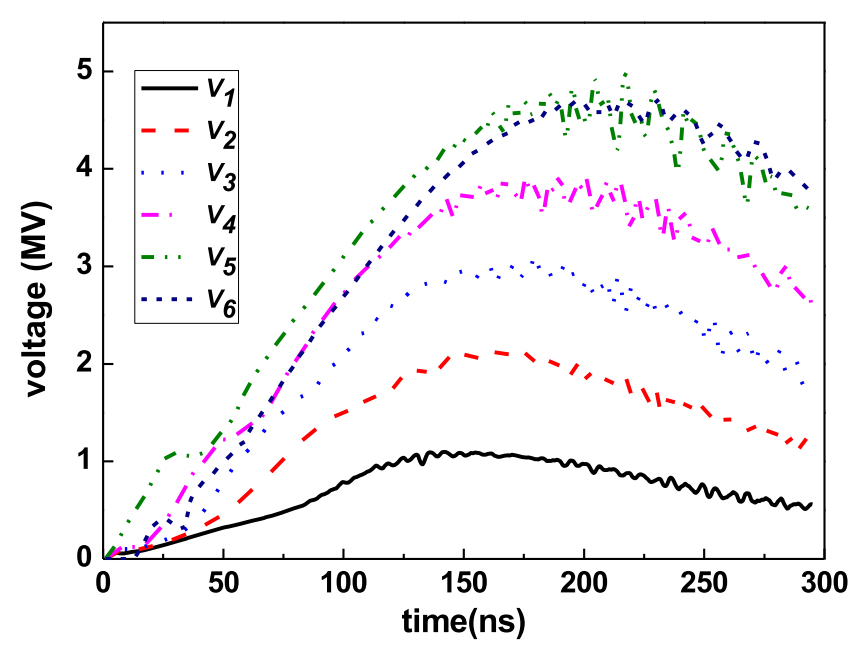

FIG. 4. Anode to cathode voltages obtained from PIC simulation. The voltages were observed after each LTD group and after the final conical line for a self-limited magnetic insulation. Results indicated voltage could be effectively summed up and a maximum value of 4.6 MV could be obtained at the final MITL. 


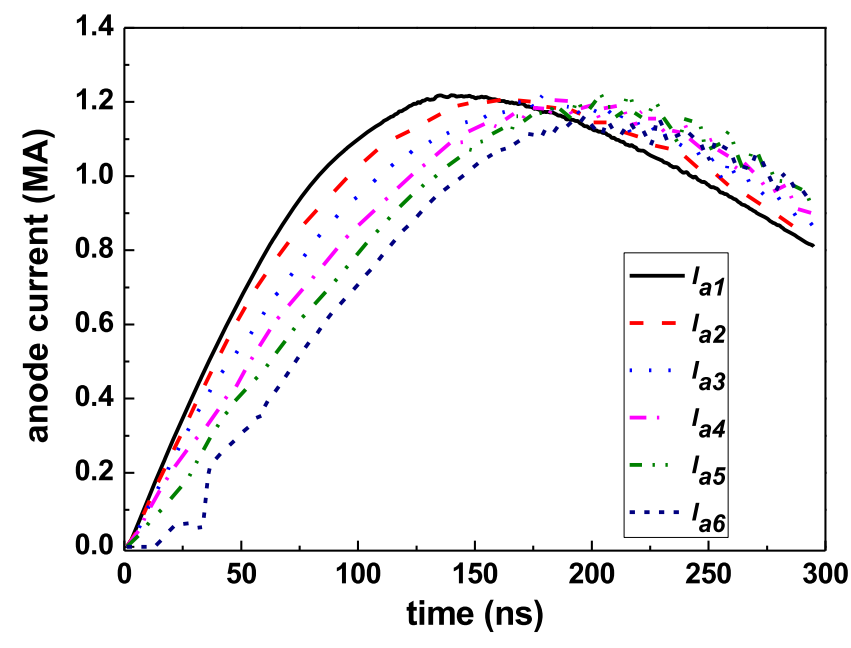

FIG. 5. Anode currents obtained from PIC simulation. The currents were observed at anode after each LTD group and after the final conical line for a self-limited magnetic insulation. The maximum current after each group was above 1.1 MA, slightly higher than the designed value. The almost constant anode current indicated magnetic insulation was well maintained.

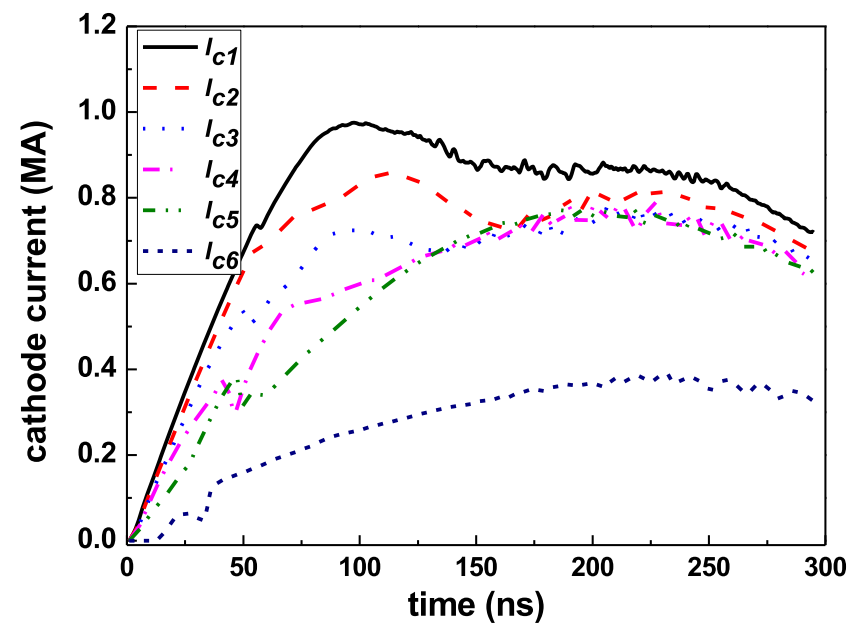

FIG. 6. Cathode currents obtained from PIC simulation. The currents were observed at cathode after each LTD group and after the final conical line for a self-limited magnetic insulation. The declined trend when approaching load indicated more and more current became electron current and flew in the anodecathode gap.

Magnetic cores were used to prevent current loss to the cavity shell. The volt-second product of the cores within a single cavity is greater than $28 \mathrm{mV} \cdot \mathrm{s}$, which ensures the core material undergo without saturation. The equivalent loss resistance of core was estimated to be $2.8 \Omega$, which is much greater than the matched impedance of the cavity $(90 \mathrm{~m} \Omega)$. This ensures the majority of current can flow to the secondary. The diameter and height of the cavity are 2.9 and $0.27 \mathrm{~m}$, and the inner and outer diameters of magnetic core are 1.47 and $1.66 \mathrm{~m}$, respectively. The diameter of the

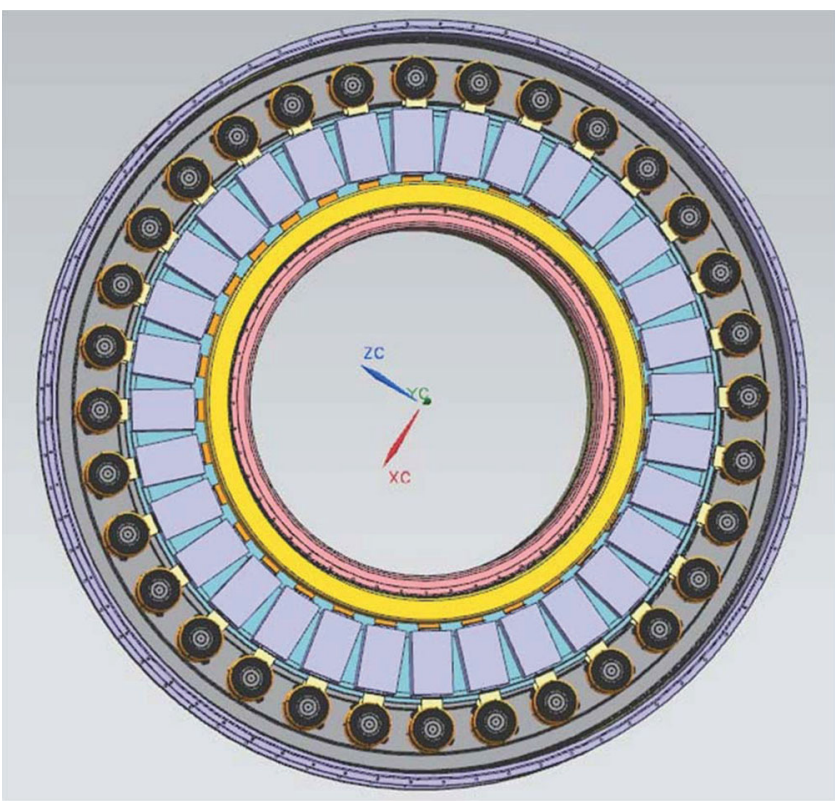

FIG. 7. Schematic of the LTD layout in M-50. The top flange was removed to show the inside. The 32 bricks were placed in spoke manner. Magnetic cores (yellow ring) prevent current losing to the ground but to secondary of the IVA.

output electrode is $1.2 \mathrm{~m}$, which is also the diameter of the outer electrode in IVA.

\section{M-50 layout}

M-50 will be linearly positioned on 40-meter rails. All 50 cavities were divided into five groups. Each group has ten cavities, and the length is about $2.7 \mathrm{~m}$. In each group, the diameter of the IVA cathode keeps constant. As shown in Fig. 8(a) with 3D schematic, each group has an independent fixing structure to make sure the cavities could be tightly compressed, well electrically connected and effectively vacuum sealed. The fixing structure has wheels and can be moved back and forth on rails. Between adjacent groups, a connecting tube (CT) with a length of $0.8 \mathrm{~m}$ is used to accommodate the vacuum pumps, electrical parameter probes and assembling operation. The tube is connected to the grounded shell of cavities and forms an anode segment of the voltage adder. After the final cavity group, three extended transmission line segments are used to connect to the load. The first segment has the same electrode radii as IVA and is in constant impedance. The length is $1.0 \mathrm{~m}$. The second segment is in a conical shape. The length is $1.5 \mathrm{~m}$, and the inner and outer electrode radii at the smaller end are the 20 and $18 \mathrm{~cm}$, respectively, which are identical with the $12 \mathrm{~m}$ MITL. The final segment is a coaxial line with a length of $0.5 \mathrm{~m}$. At the end of the final extended segment, there connects a planar electron diode, which serves as an adjustable load for module testing. 


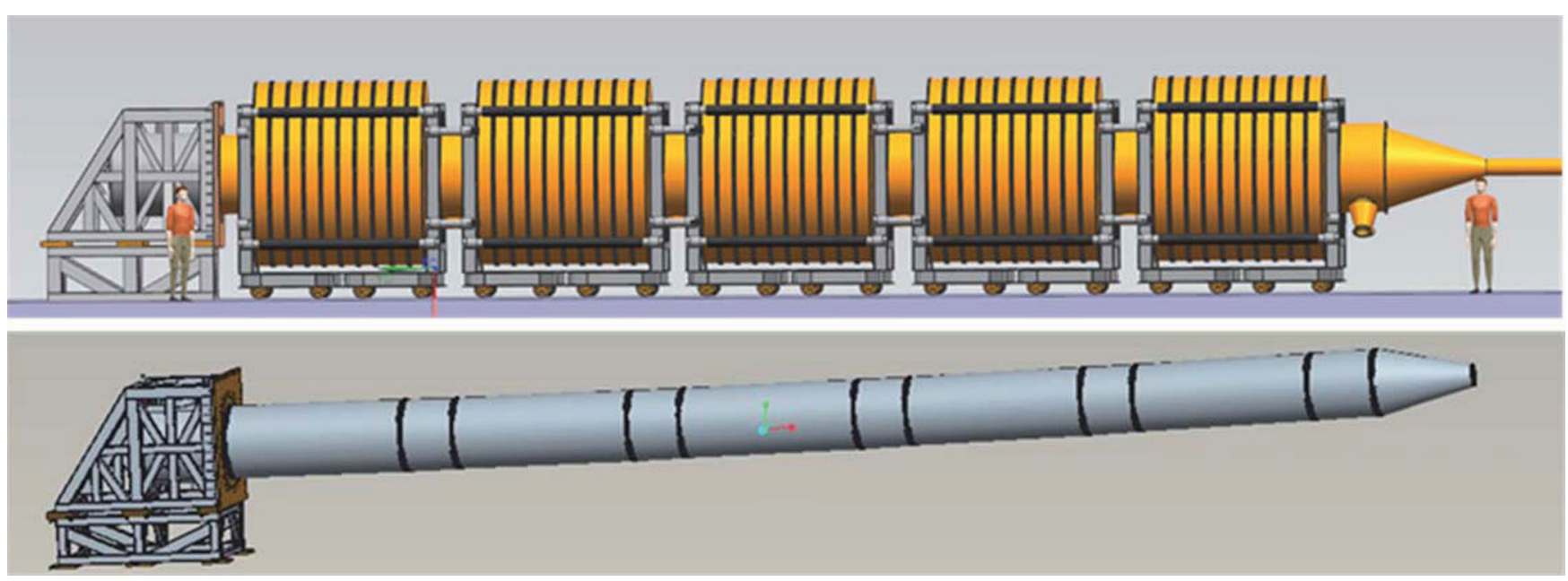

FIG. 8. Schematic of M-50 and the inner stalk of the IVA. The 50 LTD cavities are divided into five groups. Each group has independent fixing structure. The lower picture shows the inner stalk of the IVA, which is fixed to the base shelf at the left and kept free of support at the other end.

The first cavity group (leftmost) and the IVA cathode are fixed to a base shelf, which is firmly inserted into the ground and works as the support. The cathode stalk, which penetrates all cavities, is also fixed to the base shelf at the left end and keeps other positions free of any support. Since the secondary of IVA is magnetically insulated, though in principle, high impedance structural support to the inner electrode could be introduced in physics [37], no practical solution is available so far. Otherwise, loss of current or failure of magnetic insulation may happen near the support. Meanwhile, in order to avoid asymmetry induced loss, the inner and outer electrode of the MITL should keep coaxial and the gap should not be misaligned too much. So the MITL inner electrode is cantilevered from one end and must be accurately aligned within the anode tube during the experimental shot.

M-50 is generally a low impedance system whose nominal output impedance is $4.5 \Omega$, so the gap between anode and cathode in IVA is just a few centimeters. Therefore, we set the misalignment criteria of the center line to be $2 \mathrm{~mm}$. In order to achieve the goal, deflection due to gravity should be compensated. Detailed analysis in structural mechanics was conducted. The actual gravity of the cantilever is considered. Optimizations in material, structure, and compensation scheme were carried out to ensure enough strength and acceptable deformation. On the base of IVA physical design and after many iterative runs, the engineering design of the base shelf and the inner stalk were finished.

\section{Engineering consideration of the base shelf and the inner stalk $[49,50]$}

To make sure the whole facility is reliably fixed and is immune from external disturbance, M-50 should be fixed to a solid base. In Fig. 8, the base shelf at leftmost is stuck into the ground, and the outer and inner electrodes of the voltage adder are both fixed to this shelf. The shelf consists of three parts, a base, a shelf and a connecting chamber. The base and the shelf are fabricated with construction steel Q345. The connecting chamber is fabricated with stainless steel $0 \mathrm{Cr} 18 \mathrm{Ni}$, which is connected to the IVA electrodes. The total weight for the whole base shelf is about $7,000 \mathrm{~kg}$, and the overall dimension is $2,850 \mathrm{~mm}$ in height, $2,000 \mathrm{~mm}$ in width and 2,000 $\mathrm{mm}$ in length. The inner stalk is fabricated with aluminum alloy 7075 . The wall thickness is $10 \mathrm{~mm}$. The total weight is about $2,400 \mathrm{~kg}$.

Stress analysis and mechanical design were conducted by taking all load into consideration. The equivalent gravity from this stalk is $20000 \mathrm{~N}$, and the action point is $10 \mathrm{~m}$ from the connection plane. The shelf gravity itself was taken into consideration as well. Results show the maximum stress and deformation are $135 \mathrm{MPa}$ and $0.38 \mathrm{~mm}$, respectively. The stress is well below the material limitation and the deformation is also acceptable. The design is structurally safe. Figures 9 and 10 show the stress distribution and deformation distribution of the shelf, respectively, when the IVA stalk is connected to it.

Like other MIVA facilities, one of the engineering challenges is to make the cantilever stalk straight enough and within acceptable misalignment. The difficulties come from its large size, length, weight, and small A-K gaps. The most effective method is to introduce a reversed deflection in advance based on precise stress analysis and mechanics computation. The reserved deflection should be perfectly compensated by the gravity.

Figure 11 schematically explains the principle of deflection compensating for cantilever stalk. When fixing a segmental stalk in position and free of any support except at the root, the central line (blue dashed line) of the stalk will deviate from the horizon line due to gravity, and 


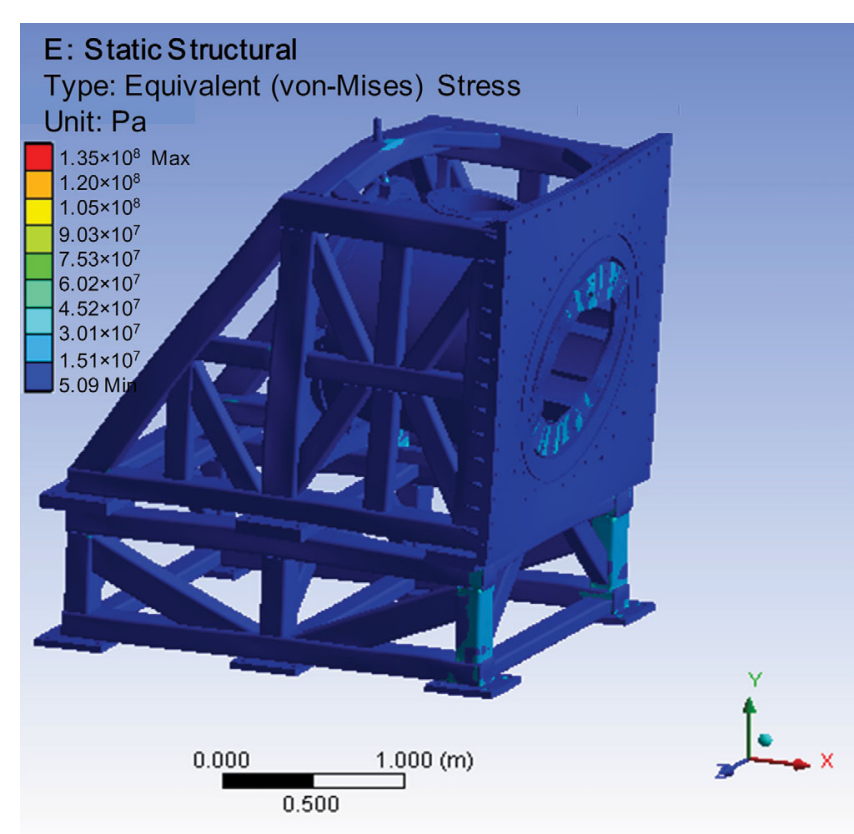

FIG. 9. Stress distribution computation and analysis when taking all load into consideration. Results show a maximum stress of $135 \mathrm{MPa}$ presents on the structure, which is within the material limit and the design is structurally safe.

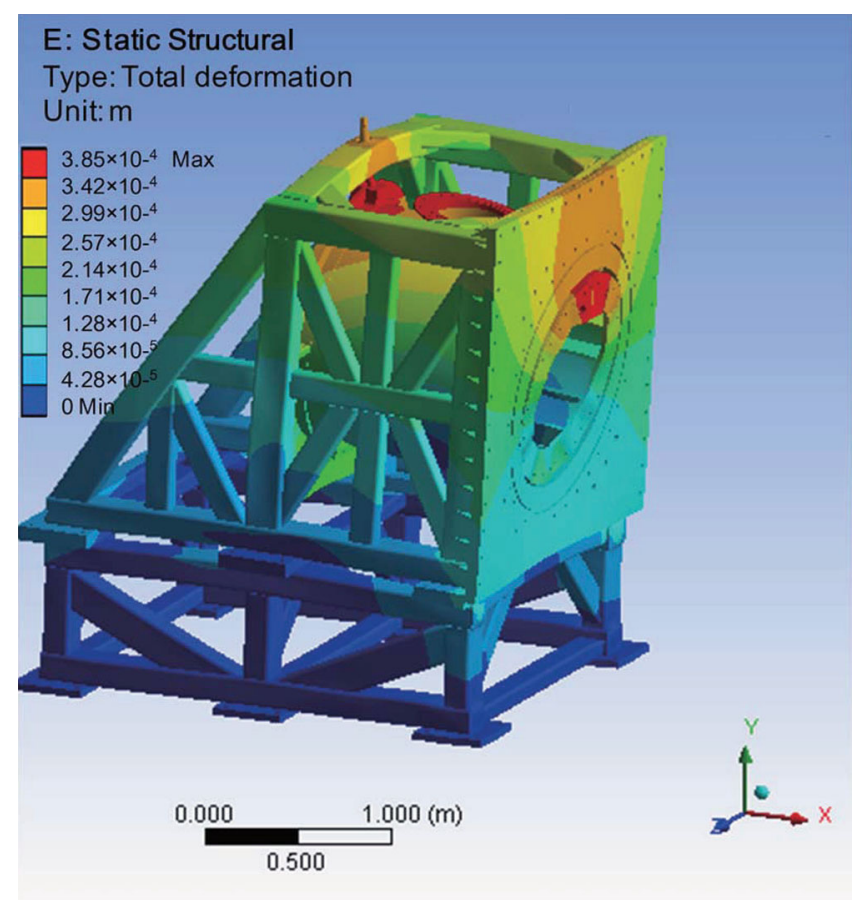

FIG. 10. Deformation distribution computation and analysis when taking all load into consideration. Results show a maximum deformation of $0.38 \mathrm{~mm}$ is induced. The deformation is acceptable in engineering.

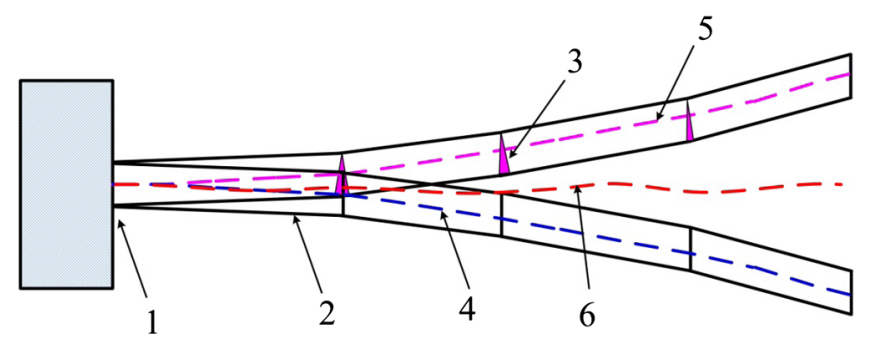

1. stalk root 2. stalk segment 3 . wedge shim

4. central line only due to gravity

5. central line only due to wedge

6. central line after compensation

FIG. 11. Principle of deflection compensating of the cantilever tube. The blue line (4) represents the central line of the cantilever when only gravity induced deflection is considered. The magenta line (5) represents the central line of the cantilever when wedge shims are introduced between segments. The red line (6) represents the final central line when the overall effect of gravity and wedge shims is considered.

the deviation grows with the distance from the root. When the root structure, stalk material, and length are known, the position of the central line could be determined by numerical calculation. To control the deviation, the stalk is divided into several segments. By introducing suitable wedge shims between segments, a deflection in the opposite direction could be introduced. Accordingly, if the gravity is ignored, the reserved deflection will cause the central line to deviate in the opposite position (magenta dashed line). After taking gravity into consideration, the reversed deflection will be perfectly compensated. Then the actual central line (red dashed line) could be aligned as expected and within limitation. The key points of this scheme are to precisely calculate the deflection of each segment, and introduce a suitable wedge to precisely precompensate the deflection. In our design, double wedge shims between two adjacent stalk segments are used. By adjusting the relative angle of the two shims, the deflection of the second segment could be changed continuously. With the help of mechanics analysis, all adjusting angles and thicknesses of shims could be determined.

\section{E. The trigger system}

The LTD system has many more switches to be triggered than traditional schemes whose switches have much higher current (for example hundreds of $\mathrm{kA}$ in $\mathrm{Z}$ and PTS) than those used in LTD. For M-50, switches in each LTD cavity are simultaneously triggered by four trigger pulses. Therefore, a total of 200 trigger pulses are needed. These pulses should be synchronized and triggered with suitable time sequence which is determined by the voltage adder. 


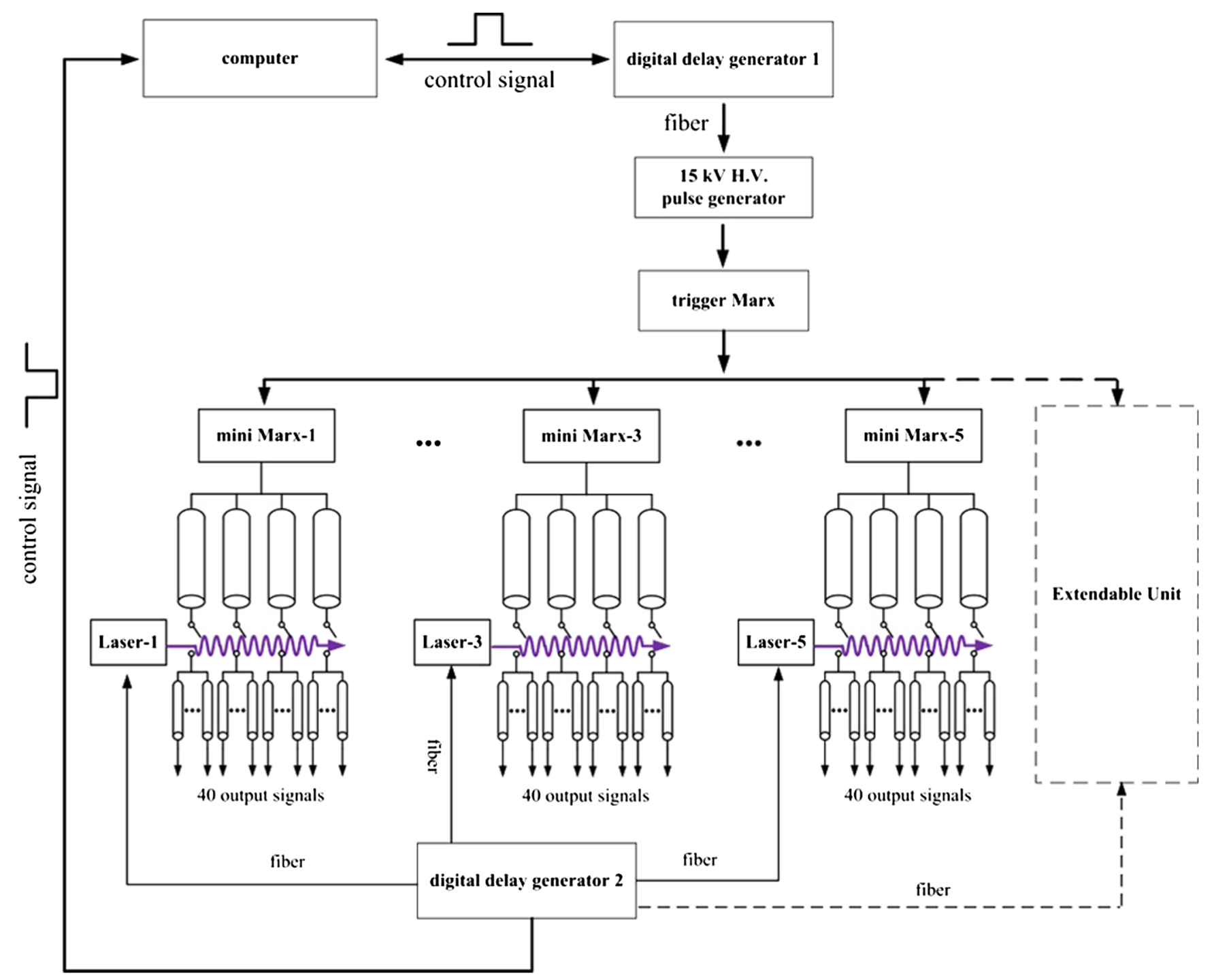

FIG. 12. Schematic of the extendable trigger system. Each unit has four pulse forming lines, four laser triggered gas switches and 40 trigger outputs. The system is extendable when a trigger unit is added to the system. The unit is controlled by a voltage pulse from the upper-level trigger Marx.

An extendable trigger system is used in M-50, whose units can be extended to accommodate future potential upgrading. Figure 12 shows the topology of the extendable trigger system [48]. The system has five basic trigger units, each of which consists of a mini-Marx generator, four water pulse forming lines, four laser triggered gas switches and 40 output cables. The output pulses from the same unit are used to trigger the LTD cavities in a group. In the upper level, before the five mini-Marx generators, there is another trigger Marx, which triggers the mini-Marx and is controlled by $15 \mathrm{kV}$ pulse from a commercially available pulse generator. The time sequence of laser and the $15 \mathrm{kV}$ pulser is controlled by two digital delay generators, which are controlled by the general control signal from computer. The advantages of this trigger scheme are simple in topology, low jitter, and extendable. The successful application of laser triggered gas switches in many facilities supports this design.
Figure 13 shows the layout of a trigger unit. A mini-Marx locates in the oil tank. The equivalent capacitance and inductance are $500 \mathrm{nF}$ and $350 \mathrm{nH}$, respectively. The conduction of the gas switch is controlled by an external voltage pulse from upper-level trigger Marx. Four $7.5 \Omega / 75$ ns water insulated pulse forming lines are connected to the output of the mini-Marx. Ten output cables, whose impedance is $75 \Omega$, are connected to each of the pulse forming lines after the laser triggered gas switch. The switch is externally triggered by a $266 \mathrm{~nm}$ laser beam, working at the fourth harmonic of a YAG laser. The output beam is split into four minibeams and each triggers one of the four switches in the trigger unit. This ensures the four switches being triggered simultaneously, and the cable outputs have low jitter. When the mini-Marx is charging to $\pm 60 \mathrm{kV}$, the pulse forming line could be charged to $200 \mathrm{kV}$ and $100 \mathrm{kV}$ voltage can be obtained on matched load; when 


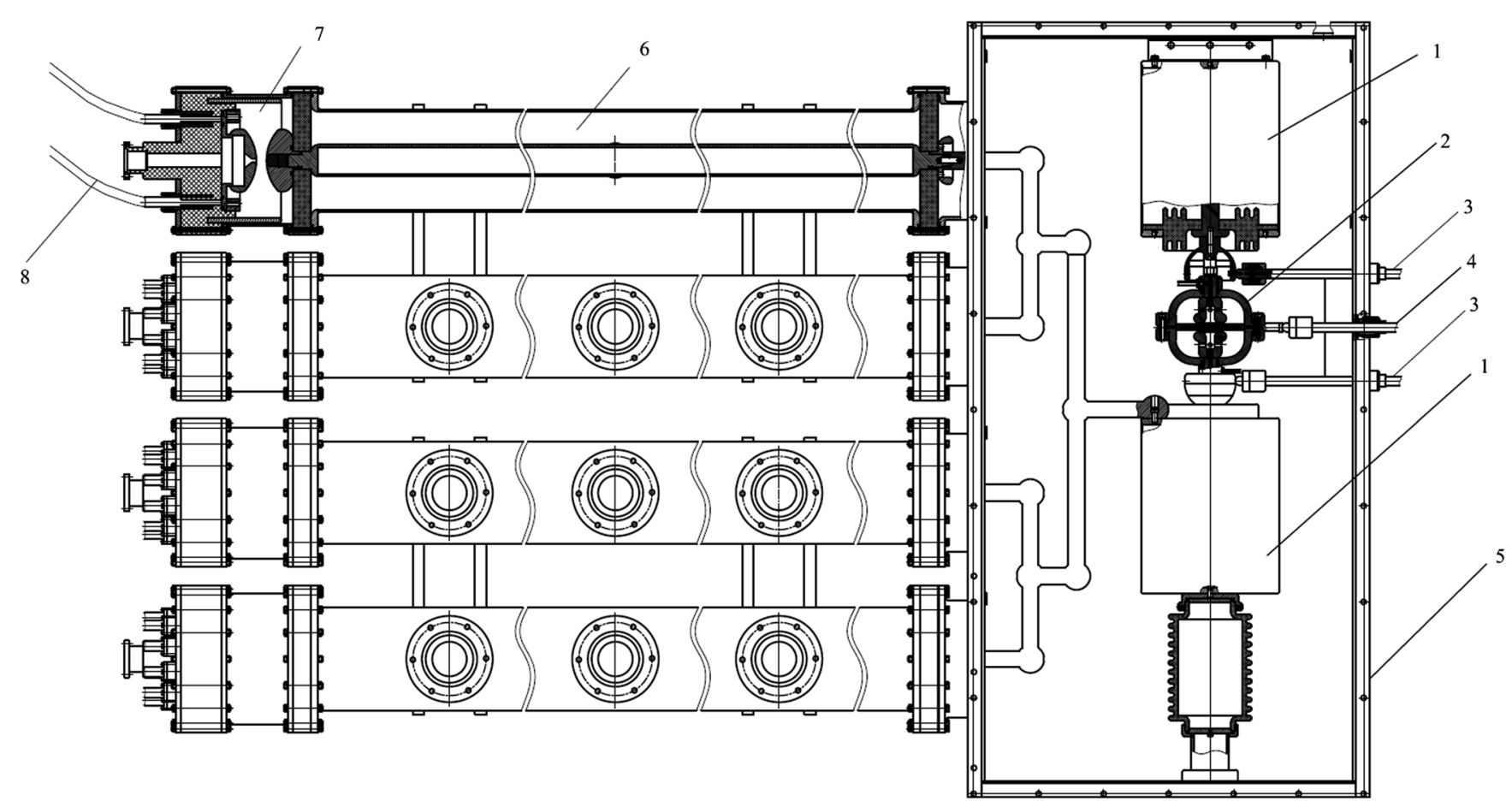

FIG. 13. Layout of a trigger unit, which includes a mini-Marx generator (5), four pulse forming lines (6), four laser triggered gas switches (7), and forty output cables (8). In the mini-Marx, there are two capacitors (1), an electrically triggered gas switch (2). The cables (3) are connected to positive/negative chargers, and cable (4) is connected to trigger.

the cable is connected to switch electrode, the 10\%-90\% voltage rising time is no more than $30 \mathrm{~ns}$.

\section{PROJECT PROGRESS}

\section{A. LTD cavities}

The project of M-50 started in 2014, but the development of LTD technology has proceeded for a decade. So far, the cavity and all related component technologies have been successfully demonstrated.

Since 2005, four types of multi-gap gas switches have been developed, which differ in gap number and length, electrode material and supporting structure. Depending on the cavity it is used, the maximum switch current is different. In the cavity of M-50, the type 4 gas switch (Fig. 14) is used. It has four gaps and a total gap length of $30 \mathrm{~mm}$. The intermediate electrodes are made of stainless steel and are supported to the switch wall by metallic balls. Corona discharge needles are used to grade the field and voltage distribution between electrodes. The switch uses dry air as insulation gas. The hold-off voltage is $200 \mathrm{kV}$ and maximum current is $40 \mathrm{kA}$. The switch jitter is about $1.6 \mathrm{~ns}$ under a working ratio of $65 \%$, when triggered by a $90 \mathrm{kV} / 15 \mathrm{~ns}$ voltage.

The uniformity of switches in a single LTD cavity has been experimentally tested and observed (Fig. 15). Fiber probes were used to collect the light signals of switches during the discharge. A CCD camera was used to record the light at the other end of the fiber. If the switch is successfully triggered, a light spot could be recorded. The CCD image could not give the temporal information on conduction, but the jitter or time variation of the triggered switches could be estimated the output current. If the triggered timing spreads in a wide range, the current rising

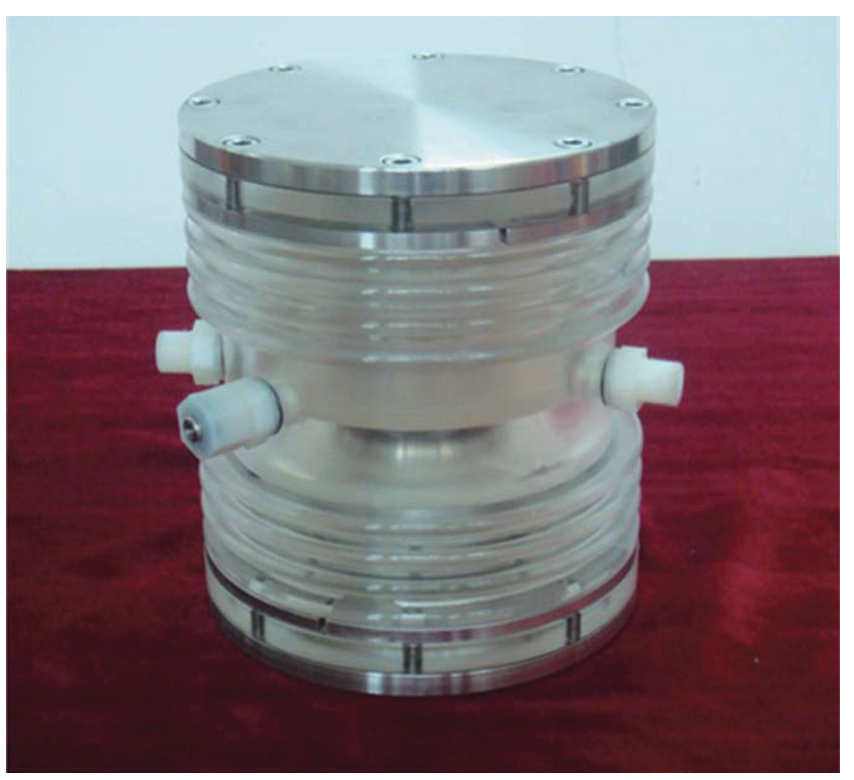

FIG. 14. Photo of the type 4 switch used in M-50. It has four gaps and a total gap length of $30 \mathrm{~mm}$. Dry air is used as insulation gas. 


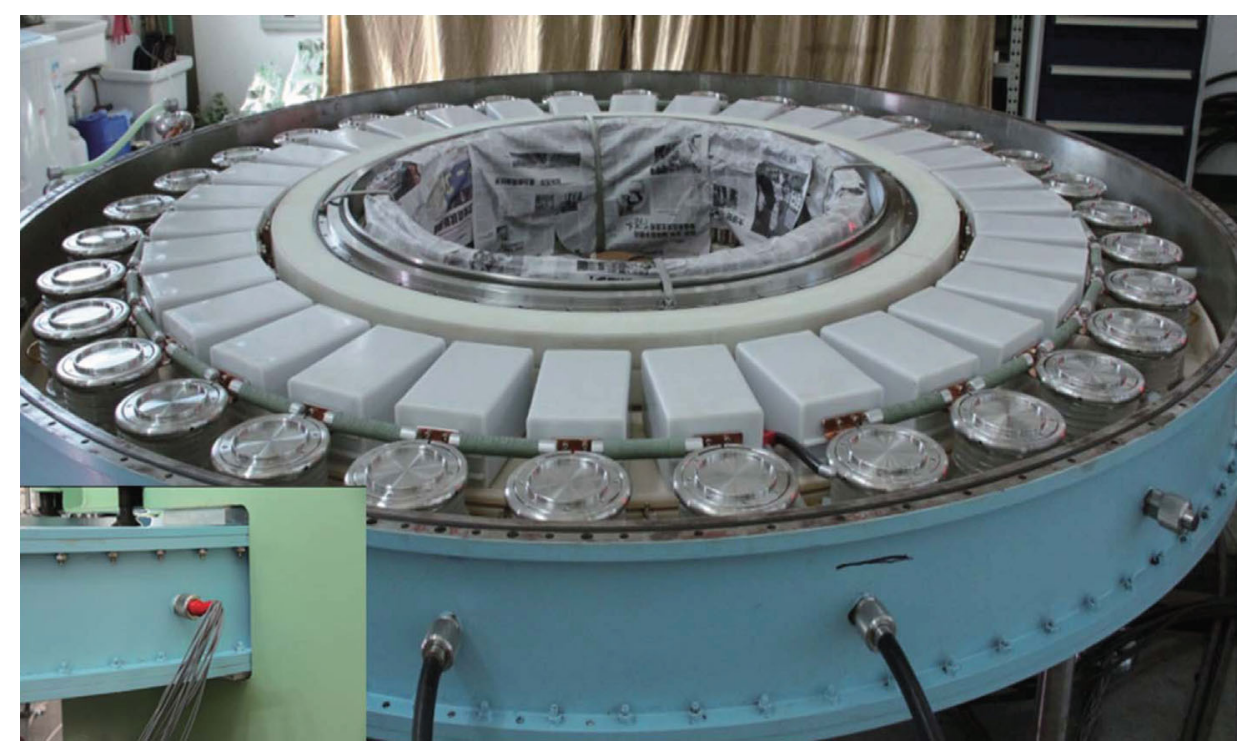

FIG. 15. Photo of the LTD cavity which is under testing. The subfigure shows the entrance into the cavity for fibers, which were used to monitor the light emission from switches.

time will be longer and the amplitude will be lower. Results show all 32 switches in the cavity could be well triggered and the cavity performs as expected. Figure 16(a) shows the recorded image by $\mathrm{CCD}$ which indicates all switches were triggered and closed, and Fig. 16(b) shows a typical comparison of cavity currents from experiment and from circuit simulation. The comparison indicates the LTD performance met the expectation. When the charging voltage was $\pm 90 \mathrm{kV}$, the output current was $1054 \mathrm{kA}$, and the $10 \%-$ 90\% rising time was $123 \mathrm{~ns}$, and the full width at half maximum (FWHM) of the current pulse was $310 \mathrm{~ns}$.

So far as this paper is written, tests to all 50 cavities of M-50 have finished under controlled conditions. The charging voltage in the test was $\pm 90 \mathrm{kV}$ and the switch working ratio was $70 \%$. To accommodate laboratory arrangement and to facilitate operation, a simpler trigger with 5 output was used. The trigger voltage was about $90 \mathrm{kV}$ and the rising time was $30 \mathrm{~ns}$. The maximum current, current rising time and pulse FWHM for each numbered cavity were monitored and recorded.

Figure 17 shows the statistical plot of performance from the tests. The horizontal axis represents the cavities number. The maximum current, rising time and FWHM for each of the 50 cavities are presented with scatters. The averaged values of each parameter for all 50 cavities are shown with dashed lines. It can be seen that the maximum

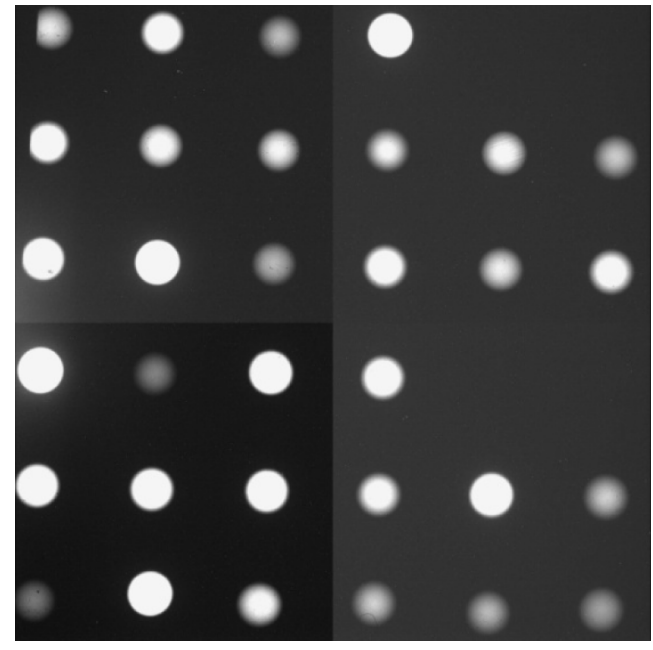

(a)

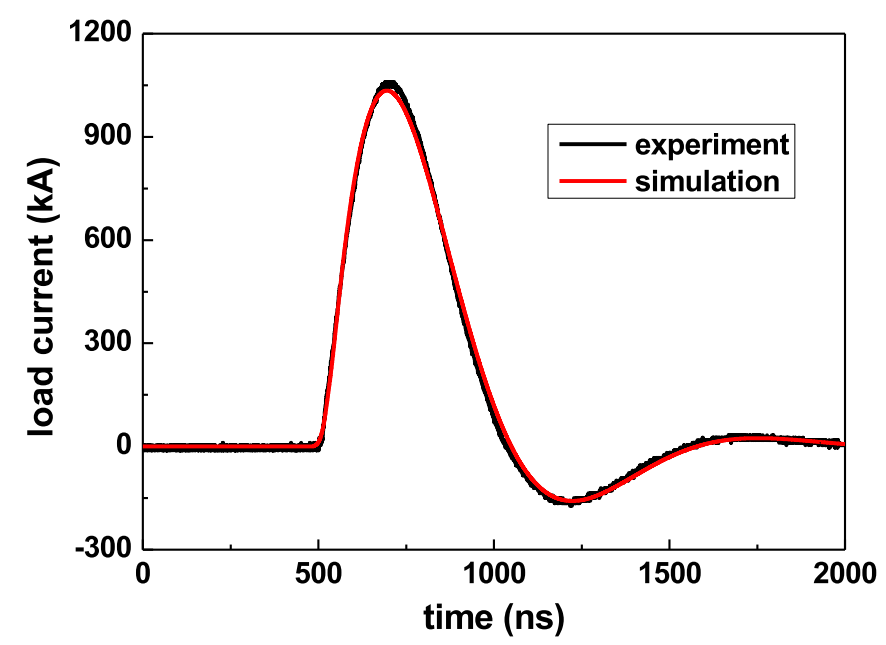

(b)

FIG. 16. (a) Image of 32 light spots recorded by CCD, which indicates all switches were closed. (b) Comparison of current pulses from experiment and from circuit simulation, indicating the performance meets expectation. 


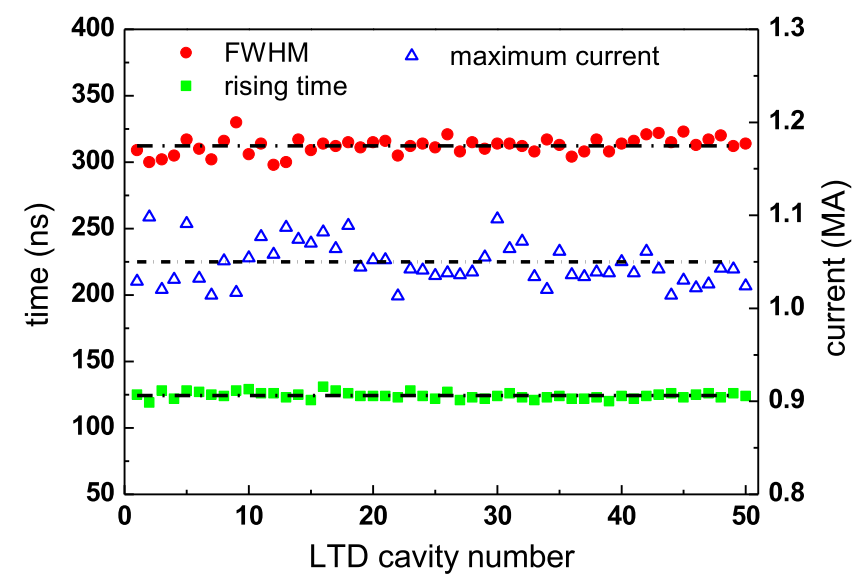

FIG. 17. Parameter statistics for 50 LTD cavities. The horizontal axis represents the cavities number and the vertical axis represents the recorded parameters. The maximum currents are shown by the blue triangle; the rising times are shown by the green square, and the FWHMs are shown by red spots. The dashed lines around scatters represent the averaged values.

currents mainly locate within 1.0 to $1.1 \mathrm{MA}$, with an average value of $1047 \mathrm{kA}$ and a standard deviation of $23 \mathrm{kA}$; the averaged current rising time is $124 \mathrm{~ns}$, with a standard deviation of $2.5 \mathrm{~ns}$; the averaged FWHM is $312 \mathrm{~ns}$, with a standard deviation of $6.4 \mathrm{~ns}$. The results indicate the cavities have satisfactory uniformity.

\section{B. Trigger system}

The first unit (Fig. 18) of the extendable trigger system has been finished and tested. More trigger units are being constructed. The output voltage at the end of the trigger cable was measured when the cable was connected to a matched load or to the trigger electrode of the switches. When the mini-Marx was charged to $\pm 60 \mathrm{kV}$, the voltage obtained on a matched load was about $103 \mathrm{kV}$, and the

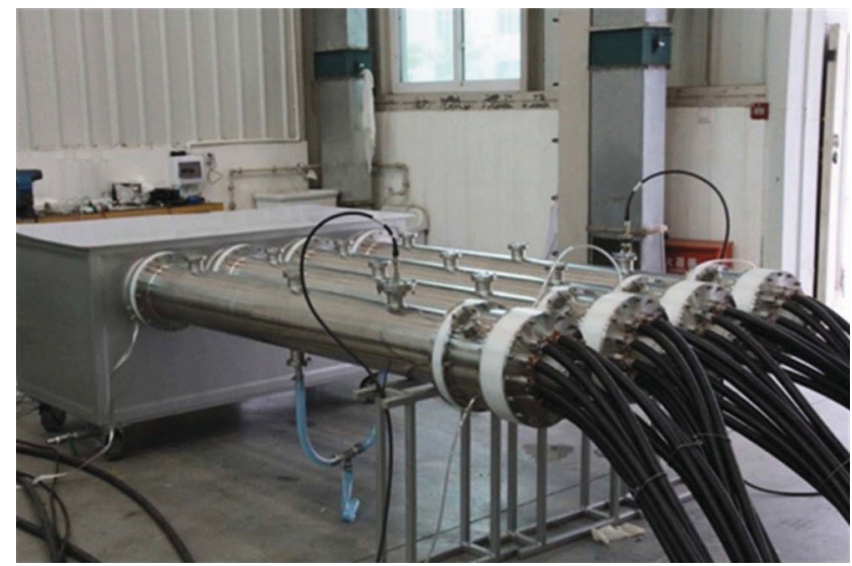

FIG. 18. The first unit of the extendable trigger system. The unit has 40 outputs, and every ten outputs from the same output are controlled by a laser trigger switch.

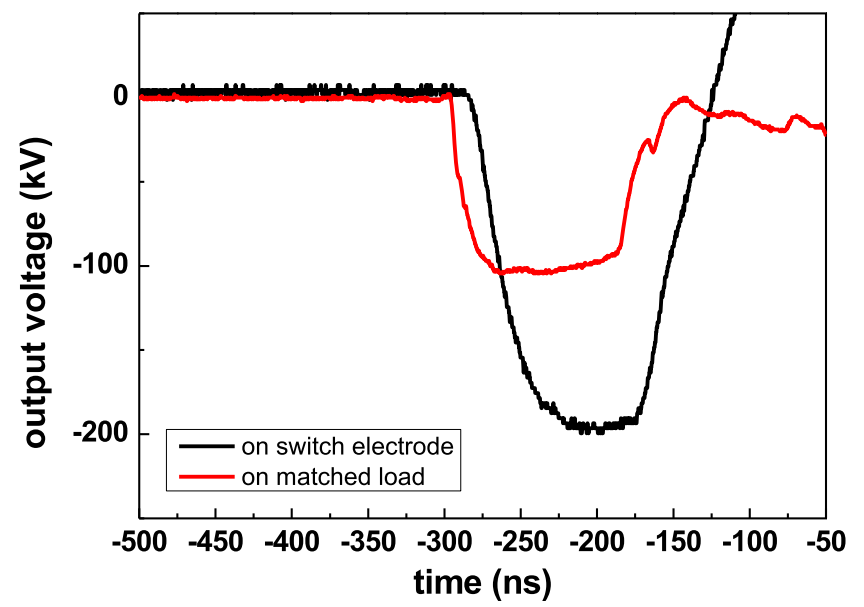

FIG. 19. Comparison of trigger output at the cable end from testing. A voltage with a maximum of $103 \mathrm{kV}$, rising time of $20 \mathrm{~ns}$ is obtained on a matched load. On the electrode of the switch, the maximum is $195 \mathrm{kV}$, and the rising time is $40 \mathrm{~ns}$.

10\%-90\% rising time was about $20 \mathrm{~ns}$. The jitter for the 40 voltage signals was less than $1 \mathrm{~ns}$. Much higher voltage was obtained when the cable was connected to the switch electrode, which was mainly due to reflection from a high impedance load. The voltage amplitude was about $195 \mathrm{kV}$, and the 10\%-90\% rising time was 40 ns (Fig. 19).

\section{IVA}

For the IVA inner stalk, all the segments and base shelf had been fabricated. Figure 20 shows the cantilever photo

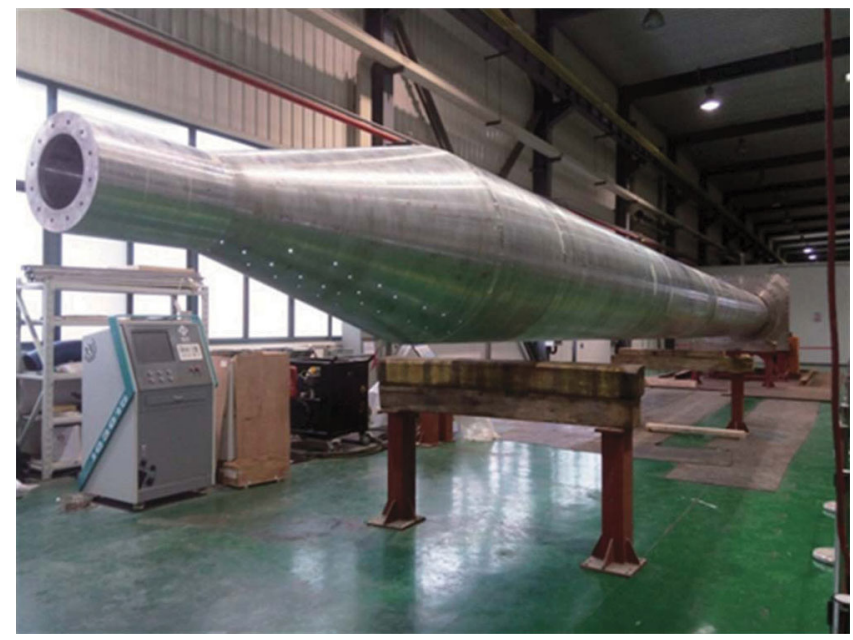

FIG. 20. The stalk cantilever after the assembling was finished. The stalk was only fixed to a replaced base at the furthest end. The gravity-induced deflection was well compensated by the wedge shim between adjacent segments. Angles and thickness of each wedge shim were set to the determined value. A maximum eccentricity of $1.48 \mathrm{~mm}$ was achieved according to laser tracking and positioning system. 
after the assembling. The stalk was assembled to a replaced base according to the precompensation scheme, in which the angle and thickness of each wedge shim were determined by numerical analysis. The spatial positions of the stalk were measured with a laser tracking and positioning system. Then the real axis of the stalk could be fitted and the deviation from ideal axis could be evaluated. The maximum eccentricity achieved during the assembling was $1.48 \mathrm{~mm}$, which met the deviation criteria of the design. Less eccentricity is possible if further adjustment is applied.

\section{CONCLUSIONS}

After a decade of involving into LTD investigation, a 50-cavity module project of MA LTD was proposed and is being carried out at CAEP. The module project, which is named M-50, aims to integrate LTD related technology and to demonstrate the engineering feasibility at facility scale. M-50 is one of the full-scale modules for future fusion accelerator.

In this paper, the conceptual design for future fusion accelerator was described, from which M-50 was proposed and the module parameters were determined. The physical and engineering design of M-50, including MIVA physical design, LTD cavity design, facility layout, inner stalk engineering and the trigger system were introduced. The up-to-date project progress was briefly described. The component technology, cavity, trigger unit, and the engineering solution for $20-\mathrm{m}$ cantilever were all tested and have reached expectations.

There are still lots of jobs ahead before the installation of M-50, including cavity group testing, trigger unit integration, affiliated system (such as pumping system, water system, gas system, safety and protection system) and house preparation. The most recent experimental demonstration for the first group of ten cavities shows positive progress in voltage adder, which will be reported in following publications.

After the installation of the M-50, more investigations are already in schedule, two of which are related with the MITL and the power flow. As described in the conceptual design, before connecting to the central convergence structure, a 12-m long coaxial MITL will be used in each module. The loss and the sharpen effect of current pulse in 12-m MITL should be tested to estimate the module output. Another issue is the influence of reversal voltage on LTD performance. Since the LTD cavities are directed coupled to load, for low impedance load, reversal voltage may be induced, which will change the electron emission and thus the power flow in MITL. Since directly coupled, the reversal voltage is harmful for the capacitor too. If the reversal ratio is too high, the lifetime of the capacitor will decrease seriously. The extent of the reversal voltage and its influence will be experimentally investigated.
[1] R. B. Spielman et al., Phys. Plasmas 5, 2105 (1998).

[2] D. D. Ryutov, M. S. Derzon, and M. K. Matzen, The physics of fast Z pinches, Rev. Mod. Phys. 72, 1 (2000).

[3] R. Paul Drake, High Energy Density Physics-Fundamentals, Inertial Fusion, and Experimental Astrophysics (Springer-Verlag, Berlin, 2006).

[4] M. Keith Matzen et al., Pulsed-power-driven high energy density physics and inertial confinement fusion research, Phys. Plasmas 12, 055503 (2005).

[5] S. A. Slutz, M. C. Herrmann, R. A. Vesey, A. B. Sefkow, D. B. Sinars, D. C. Rovang, K. J. Peterson, and M.E. Cuneo, Pulsed-power-driven cylindrical liner implosions of laser preheated fuel magnetized with an axial field, Phys. Plasmas 17, 056303 (2010).

[6] M. R. Gomez et al., Experimental Demonstration of Fusion-Relevant Conditions in Magnetized Liner Inertial Fusion, Phys. Rev. Lett. 113, 155003 (2014).

[7] T. W. Sanford, R. E. Olson, R. L. Bowers, G. A. Chandler, M. S. Derzon, D. E. Hebron, R. J. Leeper, R. C. Mock, T. J. Nash, D. L. Peterson, L. E. Ruggles, W. W. Simpson, K. W. Struve, and R.A. Vesey, Z-Pinch-Generated X Rays Demonstrate Potential for Indirect-Drive ICF Experiments, Phys. Rev. Lett. 83, 5511 (1999).

[8] R. B. Spielman and J. S. De Groot, Z pinches-A historical view, Laser Part. Beams 19, 509 (2001).

[9] E. V. Grabovskii, Dense Z-Pinches: 5th International Conference on Dense Z-Pinches, in Proceedings of a Conference held 2002 in Albuquerque, New Mexico, AIP Conference Proceedings, Vol. 651, edited by J. Davis, C. Deeney, and N. R. Pereira (American Institute of Physics, New York, 2002), pp. 3-8.

[10] X. D. Ren, X. B. Huang, S. T. Zhou, S. Q. Zhang, J. Li, L. B. Yang, and P. Li, Acta Phys. Sin. 58, 7067 (2009) [in Chinese].

[11] X.-B. Huang, S.-T. Zhou, J.-K. Dan, X.-D. Ren, K.-L. Wang, S.-Q. Zhang, J. Li, Q. Xu, H.-C. Cai, S.-C. Duan, K. Ouyang, G.-H. Chen, C. Ji, B. Wei, S.-P. Feng, M. Wang, W.-P. Xie, J.-J. Deng, X.-W. Zhou, and Y. Yang, Preliminary experimental results of tungsten wire-array Z-pinches on primary test stand, Phys. Plasmas 22, 072707 (2015).

[12] W. A. Stygar et al., Theoretical z-pinch scaling relations for thermonuclear-fusion experiments, Phys. Rev. E 72, 026404 (2005).

[13] P. Xianjue and W. Zhen, Concept research on Z-pinch driven fusion-fission hybrid reactor, High Power Laser Part. Beams 26, 090201 (2014) (in Chinese).

[14] W. A. Stygar, M. E. Cuneo, D. I. Headley, H. C. Ives, R. J. Leeper, M. G. Mazarakis, C. L. Olson, J. L. Porter, T. C. Wagoner, and J. R. Woodworth, Architecture of petawattclass z-pinch accelerators, Phys. Rev. ST Accel. Beams 10, 030401 (2007).

[15] S. A. Slutz, W. A. Stygar, M. R. Gomez, K. J. Peterson, A. B. Sefkow, D. B. Sinars, R. A. Vesey, E. M. Campbell, and R. Betti, Scaling magnetized liner inertial fusion on Z and future pulsed-power accelerators, Phys. Plasmas 23, 022702 (2016).

[16] S. A. Slutz, Scaling of magnetized inertial fusion with drive current rise-time, Phys. Plasmas 25, 082707 (2018).

[17] R. B. Spielman, F. Long, T. H. Martin, J. W. Poukey, D. B. Seidel, W. Shoup, W. A. Stygar, and D. H. McDaniel, 
PBFA II-Z: A 20-MA driver for Z-pinch experiments, in Proceedings of the 12th IEEE International Pulsed Power Conference Albuquerque, New Mexico, 1995, edited by W. Baker and G. Cooperstein, pp. 396.

[18] J. Deng et al., From concept to reality-A review to the primary test stand, and its preliminary application in high energy density physics, Matter Radiat. Extremes 1, 48 (2016).

[19] V. V. Aleksandrov, E. V. Grabovski, A. N. Gribov, G. M. Oleinik, A. A. Samokhin, and P. V. Sasorov, Transportation of an electromagnetic pulse to the load in the Angara-5-1 facility, Plasma Phys. Rep. 34, 911 (2008).

[20] K. R. LeChien et al., 6.1-MV, 0.79-MA laser-triggered gas switch for multimodule, multiterawatt pulsed-power accelerators, Phys. Rev. ST Accel. Beams 13, 030401 (2010).

[21] H. Li, J. Deng, Y. Wang, S. Feng, W. Xie, and B. Ding, Development of a $4 \mathrm{MV}$ laser-triggered multi-stage switch, Plasma Sci. Technol. 10, 235 (2008).

[22] M. Cuneo et al., Pulsed power IFE background, phased R\&D, and roadmap, at the NAS Committee on the Prospects for ICF Energy Systems, Albuquerque, New Mexico, 2011.

[23] C. L. Olson, M. G. Mazarakis et al., Report No. SAND20070059, 2007.

[24] A. N. Bastrikov, A. A. Kim, B. M. Kovalchuk et al., Fast primary energy storage based on linear transformer scheme, in Proceedings of the 11th IEEE International Pulsed Power Conference, Baltimore, Maryland USA, 1997, edited by G. Cooperstein and I. Vitkovitsky, pp. 489-497.

[25] P. Monjaux, F. Kovacs, J. F. Léon, P. L’Eplattenier, and A. Morell, Design of a modular experiment based on LTD concept for flux compression evaluation, in Proceedings of the 13th International Conference on High-Power Particle Beams, Nagaoka, Japan, 2000.

[26] D. B. Reisman, B. S. Stoltzfus, W. A. Stygar et al. Pulsed power accelerator for material physics experiments, Phys. Rev. ST Accel. Beams 18, 090401 (2015).

[27] A. A. Kim, A. N. Bastrikov, B. M. Kovalchuk et al., $1 \mathrm{MV}$ ultrafast LTD generator, in Proceedings of 14th IEEE International Pulsed Power Conference, Dallas, Texas, 2003, edited by M. Giesselmann and A. Neuber, (2003), pp. 853-854.

[28] L. Zhou, J. Deng, C. Lin et al., Design and experiment of linear transformer driver stage, High Power Laser Part. Beams 18, 1749 (2006) [in Chinese].

[29] J. J. Leckbee, J. E. Maenchen, D. L. Johnson, S. Portillo, D. M. Vandevalde, D. V. Rose, and B. V. Oliver, Design, simulation, and fault analysis of a 6.5-MV LTD for flash x-ray radiography, IEEE Trans. Plasma Sci. 34, 1888 (2006).

[30] A. A. Kim, V. Sinebryukhov, A. N. Bastrikov et al., Design and first tests of five $100 \mathrm{GW}$ fast LTD cavities driving an e-beam diode load, in Proceedings of the 16th IEEE International Pulsed Power Conference, Albuquerque, NM (IEEE, Piscataway, NJ, 2007), pp. 144-147.

[31] T. Liang, F. Sun, A. Qiu et al., Simulation and primary experimentation of linear transformer driver module, High Volt. Eng. 33, 18 (2007).
[32] A. A. Kim, M. G. Mazarakis, V. A. Sinebryukhov, B. M. Kovalchuk, V. A. Visir, S. N. Volkov, F. Bayol, A. N. Bastrikov, V. G. Durakov, S. V. Frolov, V. M. Alexeenko, D. H. McDaniel, W. E. Fowler, K. LeChien, C. Olson, W. A. Stygar, K. W. Struve, J. Porter, and R. M. Gilgenbach, Development and tests of fast 1-MA linear transformer driver stages, Phys. Rev. ST Accel. Beams 12, 050402 (2009).

[33] L. Chen, L. J. Zhou, W. K. Zou et al., The design and the first test results of a fast LTD stage, Acta Phys. Pol. 115, 1186 (2009).

[34] M. G. Mazarakis, W. E. Fowler, K. L. LeChien et al., High-current linear transformer driver development at Sandia National Laboratories, IEEE Trans. Plasma Sci. 38, 704 (2010).

[35] J. J. Leckbee, S. R. Cordova, B. V. Oliver, T. J. Webb, M. Toury, M. Caron, R. Rosol, B. Bui, T. Romero, and D. Ziska, Linear transformer driver (LTD) research for radiographic applications, in Proceedings of the 18th IEEE International Pulsed Power Conference, Chicago IL, 2011, edited by R. D. Curry and B. V. Oliver (2011), pp. 614-618.

[36] F. Bayol, F. Cubaynes, R. Delplanque, P. Genez, C. Legras, M. Parzych, M. Toury, M. Caron, M. Mouillet, and A. A. Kim, Development of a 1 MV Ultra-Fast LTD Generator, in Proceedings of the 18th IEEE International Pulsed Power Conference Chicago, IL, 2011, edited by D. Curry and B. V. Oliver (2011), pp. 619-624.

[37] W. Zou, L. Chen, D. Liu, L. Zhang, L. Liu, L. Zhou, M. Wang, B. Wei, F. Guo, X. Wang, Y. Dai, W. Xie, and J. Deng, Investigation on high inductive helical supported magnetically insulated transmission line on a 10-stage linear transformer driver system, Phys. Rev. ST Accel. Beams 15, 110401 (2012).

[38] M. Toury, F. Cartier, P. Combes et al.,Transfer and test of a 1 MV LTD generator at CEA, in Proceedings of the 19th IEEE International Pulsed Power Conference San Francisco, CA, 2013, edited by B. V. Oliver, J. P. Verboncoeur and M. T. Crawford (2013), pp. 766-769.

[39] J. D. Douglass et al., $100 \mathrm{GW}$ linear transformer driver cavity: Design, simulations, and performance, Phys. Rev. Accel. Beams 21, 120401 (2018).

[40] I. D. Smith, Induction voltage adders and the induction accelerator family, Phys. Rev. ST Accel. Beams 7, 064801 (2004).

[41] J. W. Poukey and K. D. Bergeron, Space-charge effects in long coaxial vacuum transmission lines, Appl. Phys. Lett. 32, 8 (1978).

[42] J. M. Creedon, Relativistic Brillouin flow in the high $v / \gamma$ diode, J. Appl. Phys. 46, 2946 (1975).

[43] J. M. Creedon, Magnetic cutoff in high-current diodes, J. Appl. Phys. 48, 1070 (1977).

[44] W. Zou, B. Wei, L. Liu et al., Coaxial-conical transition in magnetically insulated transmission lines, IEEE Trans. Plasma Sci. 46, 1913 (2018).

[45] J. Zhou, D. Liu, C. Liao, and Z. Li, CHIPIC: An efficient code for electromagnetic PIC modeling, and simulation, IEEE Trans. Plasma Sci. 37, 2002 (2009).

[46] L. Liu, W. Zou, D. Liu, F. Guo, H. Wang, and L. Chen, PIC simulation of the vacuum power flow for a 5 terawatt, 
5 MV, 1 MA pulsed power system, AIP Adv. 8, 035112 (2018).

[47] L. Chen, M. Wang, W. Xie et al., Design of the linear transformer driver stage prototype for Z-pinch driver, High Power Laser Part. Beams 26, 095007 (2014) [in Chinese].

[48] L. Chen, M. Wang, W. Zou, L. Zhou, F. Guo, and W. Xie, Recent advances in fast linear transformer driver in CAEP, High Volt. Eng. 41, 1798 (2015) [in Chinese].
[49] M. Deng, K. Lian, and J. Zhu, Structure design of an ultralong and collimating cantilever tube (unpublished).

[50] L. Chen, W. Zou, L. Zhou, J. Jiang, B. Wei, A. He, M. Wang, W. Xie, J. Wang, and Y. Zhang, Design, and the first test results of ten 1 MA-LTD cavities stacked in series, in Proceedings of EAPPC and BAEMS 2018, Changsha, China, 2018, edited by J. Zhang (2018), pp. 124-127. 